\title{
Transversal Surfaces of Ruled Surfaces in the Pseudo-Galilean Space
}

\author{
By \\ Željka Milin Šipuš and Blaženka Divjak
}

(Vorgelegt in der Sitzung der math.-nat. Klasse am 22. Jänner 2004

durch das w. M. Ludwig Reich)

\begin{abstract}
In this paper we describe the transversal surfaces of ruled surfaces in the pseudoGalilean space $G_{3}^{1}$. There are three types of transversal surfaces. The obtained results can be easily transferred to the Galilean space $G_{3}$.
\end{abstract}

Mathematics Subject Classification (2000): 53A35.

Key words and phrases: Pseudo-Galilean space, Galilean space, transversal surface, ruled surface.

\section{Introduction}

In the three-dimensional Euclidean space $E^{3}$ the notion of $\gamma$-transversal surfaces was defined in the works of G. PIRONDINI and later in the works of K. GOROWARA. H. SACHS [6] studied $\gamma$-transversal surfaces, as well as $\alpha$ - and $\beta$-transversal surfaces, by means of natural invariants [4] of ruled surfaces. The same objects in simply isotropic space $I_{3}^{1}$ were studied by A. TAOUKTSOGLOU in [7] for the ruled surfaces of the most general type.

The pseudo-Galilean space $G_{3}^{1}$ is the three-dimensional real affine space with the absolute figure $\{\omega, f, I\}$, where $\omega$ is a fixed plane, $f$ a line in $\omega$ and $I$ a hyperbolic involution of the points of $f$. The absolute figure in the Galilean space $G_{3}$ has an elliptic involution instead of the 
hyperbolic involution of points of $f$. The pseudo-Galilean length of the vector $\mathbf{x}=(x, y, z)$ is given by

$$
|\mathbf{x}|= \begin{cases}x, & x \neq 0, \\ \sqrt{\left|y^{2}-z^{2}\right|,} & x=0 .\end{cases}
$$

The group of motions of $G_{3}^{1}$ is a six-parameter group given (in affine coordinates) by

$$
\begin{aligned}
& \bar{x}=a+x, \\
& \bar{y}=b+c x+y \operatorname{ch} \varphi+z \operatorname{sh} \varphi, \\
& \bar{z}=d+e x+y \operatorname{sh} \varphi+z \operatorname{ch} \varphi .
\end{aligned}
$$

It leaves invariant the pseudo-Galilean length (1.1) of the vector.

The natural geometry of ruled surfaces in the pseudo-Galilean space $G_{3}^{1}$ is studied in [1] and of ruled surfaces in the Galilean space $G_{3}$ in [5]. In both spaces there exist three types of ruled surfaces among which the first type is the most general one. Our aim is to study transversal surfaces for that class of ruled surfaces.

\section{2. $\alpha$-Transversal Surfaces}

A ruled surface $\Phi$ of type $\mathrm{I}$ in $G_{3}^{1}$ is a surface parametrized by

$$
\mathbf{x}(x, v)=\mathbf{s}(x)+v \mathbf{e}(x),
$$

where $\mathbf{e}(x)=\left(1, e_{2}(x), e_{3}(x)\right)$ is a unit generator vector and $\mathbf{s}(x)=$ $(x, y(x), z(x))$ is the striction curve $s$ of $\Phi$, which means $y^{\prime} e_{2}^{\prime}-z^{\prime} e_{3}^{\prime}=$ $e_{2} e_{2}^{\prime}-e_{3} e_{3}^{\prime}$ and $x$ is the arc length on $s$.

For the natural trihedron $\mathbf{e}_{1}(x)=\mathbf{e}(x), \mathbf{e}_{2}=\mathbf{e}^{\prime}(x) / \kappa(x), \mathbf{e}_{3}(x)=$ $\left(0, e_{3}^{\prime}(x), e_{2}^{\prime}(x)\right) / \kappa(x)$, where $\kappa=\left|e_{2}^{\prime 2}-e_{3}^{\prime 2}\right|, \tau=\left(e_{2}^{\prime} e_{3}^{\prime \prime}-e_{2}^{\prime \prime} e_{3}^{\prime}\right) / \kappa^{2}$, the Frenet's formulas hold

$$
\mathbf{e}_{1}^{\prime}=\kappa(x) \mathbf{e}_{2}, \quad \mathbf{e}_{2}^{\prime}=\tau(x) \mathbf{e}_{3}, \quad \mathbf{e}_{3}^{\prime}=\tau(x) \mathbf{e}_{2} .
$$

Functions $\kappa$ and $\tau$ are the curvature and the torsion of $\Phi$.

The parameter of distribution of the surface $\Phi$ is given by

$$
\delta=\frac{\left(\mathbf{s}^{\prime}, \mathbf{e}, \mathbf{e}^{\prime}\right)}{\mathbf{e}^{\prime 2}}=\frac{\sigma}{\kappa},
$$

where $\sigma$ is the striction of $\Phi$ [1] defined as the isotropic angle between $\mathbf{e}_{1}$ and $\mathbf{e}_{3}$

$$
\mathbf{s}^{\prime}(x)=\mathbf{e}_{1}(x)+\sigma(x) \mathbf{e}_{3}(x) .
$$

The asymptotic plane $\eta$ of the surface $\Phi$ in the point $\mathbf{s}(x)$ spanned by $\mathbf{e}_{1}(x)$ and $\mathbf{e}_{2}(x)$ is an isotropic plane. Therefore we can define a 
vector $\overline{\mathbf{e}}(x)$ as the vector in $\eta$ that forms a constant isotropic angle $\alpha \in \mathbf{R}$ with $\mathbf{e}_{1}(x)$. An $\alpha$-transversal surface $\Phi_{\alpha}$ is the ruled surface whose rulings are straight lines through a striction point $\mathbf{s}(x)$ determined by $\overline{\mathbf{e}}(x)$. Therefore, $\Phi_{\alpha}$ can be parametrized as

$$
\overline{\mathbf{x}}(x, v)=\mathbf{s}(x)+v \overline{\mathbf{e}}(x),
$$

where $\overline{\mathbf{e}}(x)=\mathbf{e}_{1}(x)+\alpha \mathbf{e}_{2}(x)$. The striction point on the ruling $\overline{\mathbf{e}}(x)$ on $\Phi_{\alpha}$ is determined from the condition

$$
v_{0}=\frac{\left(\tilde{\overline{\mathbf{e}}}-\tilde{\mathbf{s}}^{\prime}\right) \cdot \tilde{\overline{\mathbf{e}}}^{\prime}}{\tilde{\overline{\mathbf{e}}}^{\prime 2}},
$$

where $\tilde{\mathbf{x}}$ denotes the canonical projection of the vector $\mathbf{x}$ on the $y z$-plane and t the pseudo-Galilean inner product which induces the length (1.1). By means of invariants $\kappa, \tau$ and $\sigma$, the previous expression can be written as

$$
v_{0}=\frac{\alpha(\kappa+\tau \sigma)}{\left|\kappa^{2}-\alpha^{2} \tau^{2}\right|} .
$$

Therefore, in the non-trivial case $\alpha \neq 0$, the striction curve $s$ of $\Phi$ coincides with the striction curve $s_{\alpha}$ of $\Phi_{\alpha}$ if and only if $\kappa+\tau \sigma=0$. Since the previous condition characterizes the striction curve as asymptotic on $\Phi$ [2], we have the following analogue of the Euclidean result [6]:

Theorem 2.1. The striction curve on every $\Phi_{\alpha}$ coincides with the striction curve $s$ on $\Phi$ if and only if $s$ is asymptotic on $\Phi$.

In simply isotropic space $I_{3}^{1}$ the previous situation never happens.

We can notice that the $\alpha$-transversal surface $\Phi_{\alpha}$ is of type I ( $s_{\alpha}$ does not lie in a pseudo-Euclidean plane and rulings are non-isotropic) if and only if

$$
1+\left(\frac{\kappa+\tau \sigma}{\left|\kappa^{2}-\alpha^{2} \tau^{2}\right|}\right)^{\prime} \neq 0 .
$$

This happens always if $s$ is asymptotic on $\Phi$. Furthermore, we assume that $\kappa_{s}^{2}=\overline{\mathbf{e}}^{\prime 2}=\left|\kappa^{2}-\alpha^{2} \tau^{2}\right| \neq 0$, that is, the surface $\Phi$ is not a surface of the constant slope equal to $\pm 1 / \alpha$. Surfaces of constant slope are skew non-conoidal ruled surfaces that close a constant angle $\vartheta$ with a pseudo-Euclidean plane. They are characterized by the condition $\vartheta^{2}=k^{2}=$ const, where $k=\tau / \kappa$ is the conical curvature of $\Phi$ [3].

The parameter of distribution of the surface $\Phi_{\alpha}$ is given by

$$
\delta_{\alpha}=\frac{\left(\mathbf{s}^{\prime}, \overline{\mathbf{e}}, \overline{\mathbf{e}}^{\prime}\right)}{\overline{\mathbf{e}}^{\prime 2}}=\frac{\alpha^{2} k+\delta \kappa}{\kappa\left|1-\alpha^{2} k^{2}\right|},
$$

where $\delta$ is the parameter of distribution of the surface $\Phi$. 
As in [6] we introduce the notion of $K$-surfaces as the surfaces of the constant parameter of distribution whose striction curve is asymptotic. The following theorem is an analogue of the theorem which holds in $E^{3}$ [6], whereas in $I_{3}^{1}$ it holds for another class of surfaces [7]:

Theorem 2.2. The only ruled $C^{3}$-surfaces whose all $\alpha$-transversal surfaces are of constant parameter of distribution are conoidal surfaces of constant parameter of distribution, ruled screw surfaces and $K$-surfaces. The constant parameter of distribution is equal for all $\alpha$-transversal surfaces if and only if $\Phi$ is a conoidal surface with constant parameter of distribution.

Proof. If we consider $\delta_{\alpha}$ as a function of $x$ and $\alpha$, then all $\alpha$-transversal surfaces of $\Phi$ have constant parameter of distribution if and only if $\partial \delta(x, \alpha) / \partial x=0$, for all $\alpha \in \mathbf{R}$. Therefore we have

$$
\alpha^{4} k^{2}\left(\kappa^{\prime} k+k^{\prime} \kappa\right)+\alpha^{2}\left(k^{\prime} \kappa-k \kappa^{\prime}-\delta^{\prime} \kappa^{2} k^{2}+2 \delta \kappa^{2} k k^{\prime}\right)+\delta^{\prime} \kappa^{2}=0 .
$$

For $\alpha=0$ it follows $\delta^{\prime}=0$, which implies the following conditions

$$
k^{2}\left(\kappa^{\prime} k+k^{\prime} \kappa\right)=0, \quad k^{\prime} \kappa-k \kappa^{\prime}+2 \delta \kappa^{2} k k^{\prime}=0 .
$$

Therefore, either $k=0$ ( $\Phi$ is conoidal) or

$$
\kappa^{\prime} k+k^{\prime} \kappa=0 .
$$

The condition (2.3) substituted in the second condition of (2.2) gives $k^{\prime}(\kappa+\tau \sigma)=0$. If $k^{\prime}=0$ then together with (2.3) and $\delta=$ const, we get $\kappa=$ const, $\tau=$ const, $\sigma=$ const. These invariants describe ruled screw surfaces. Finally, the condition $\kappa+\tau \sigma=0$ with $\delta=$ const implies that $\Phi$ is a $K$-surface.

Obviously, $\delta_{\alpha}$ does not depend on $\alpha$ if and only if $k=0$, which gives the last statement of the theorem.

The conical curvature of an $\alpha$-transversal surface $\Phi_{\alpha}$ is equal to

$$
k_{\alpha}=\frac{\left(\overline{\mathbf{e}}, \overline{\mathbf{e}}^{\prime}, \overline{\mathbf{e}}^{\prime \prime}\right)}{\left|\overline{\mathbf{e}}^{\prime}\right|^{3}}=\frac{\tau\left(1-\alpha^{2} k^{2}\right)+\alpha k^{\prime}}{\kappa\left|1-\alpha^{2} k^{2}\right|^{3 / 2}} .
$$

Theorem 2.3. The only ruled $C^{3}$-surfaces whose all $\alpha$-transversal surfaces are surfaces of constant slope (conoidal surfaces) are surfaces of constant slope (conoidal surfaces). The slope always varies with $\alpha$.

Proof. All $\alpha$-transversal surfaces are surfaces of constant slope if and only if $\partial k(x, \alpha) / \partial x=0$, for all $\alpha \in \mathbf{R}$. It follows immediately $k^{\prime}=0$. Similarly, $k_{\alpha}=0$, for all $\alpha$, if and only if $k=0$. 
Surfaces $\Phi$ and $\Phi_{\alpha}$ are never tangent to each other along the striction curve $s$ of $\Phi$ (they intersect each other transversally). Along $s$ they intersect each other under the angle

$$
\operatorname{ch} \varphi_{\alpha}=\frac{|\sigma|}{\sqrt{\left|\sigma^{2}-\alpha^{2}\right|}} .
$$

To see this, let us notice that the tangent plane of $\Phi$ along $s$ is given by

$$
\left(\mathbf{x}-\mathbf{s}(x), \mathbf{s}^{\prime}(x), \mathbf{e}(x)\right)=0,
$$

and of $\Phi_{\alpha}$ by

$$
\left(\mathbf{x}-\mathbf{s}(x), \mathbf{s}^{\prime}(x), \overline{\mathbf{e}}(x)\right)=0 .
$$

If we denote by $X, Y, Z$ the coordinates of an arbitrary point of these planes with respect to the basis $\left\{\mathbf{s}(x) ; \mathbf{e}(x), \mathbf{e}_{2}(x), \mathbf{e}_{3}(x)\right\}$, for the tangent plane of $\Phi$ we get $Y=0$ and for the tangent plane of $\Phi_{\alpha}$

$$
\alpha \sigma X-\sigma Y-\alpha Z=0 .
$$

Since the angle between two isotropic non-light planes $A_{i} X+B_{i} Y+$ $C_{i} Z+D_{i}=0, B_{1}: C_{1} \neq B_{2}: C_{2}, B_{i}^{2}-C_{i}^{2} \neq 0, i=1,2$, is equal to (see [1])

$$
\operatorname{ch} \varphi=\frac{\left|B_{1} B_{2}-C_{1} C_{2}\right|}{\sqrt{\left|\left(B_{1}^{2}-C_{1}^{2}\right)\left(B_{2}^{2}-C_{2}^{2}\right)\right|}},
$$

statement (2.4) follows immediately. For $\sigma=\alpha$ the tangent plane of $\Phi_{\alpha}$ is an isotropic light-like plane, and the angle is not defined. Therefore, the following theorem in $G_{3}^{1}$ differs from its analogue in $E^{3}$, whereas in $I_{3}^{1}$ surfaces $\Phi$ and $\Phi_{\alpha}$ always intersect each other orthogonally:

Theorem 2.4. Ruled $C^{3}$-surfaces $\Phi$ and $\Phi_{\alpha}$ intersect each other along $s$ under the constant angle if and only if the striction of the surface $\Phi$ is constant. The angle always varies with $\alpha$.

Furthermore, the asymptotic plane $Z=0$ in $\mathbf{s}(x)$ of a surface $\Phi$ and the asymptotic plane

$$
\alpha^{2} \tau X-\alpha \tau Y+\kappa Z=0
$$

in $\mathbf{s}(x)$ of a surface $\Phi_{\alpha}$ intersect each other under the angle

$$
\operatorname{ch} \phi_{\alpha}=\frac{1}{\sqrt{\left|1-\alpha^{2} k^{2}\right|}} .
$$

From the previous expression it follows: 
Theorem 2.5. The angle between the corresponding asymptotic plane of a ruled $C^{3}$-surface $\Phi$ and the asymptotic plane of its $\alpha$-transversal surface $\Phi_{\alpha}$ is constant if and only if $\Phi$ is a surface of constant slope. That angle is equal for all surfaces $\Phi_{\alpha}$ if and only if $\Phi$ is conoidal.

Finally, we can calculate the angle between the striction curve $s$ of $\Phi$ and the asymptotic plane of the surface $\Phi_{\alpha}$. The angle between a non-isotropic vector $x=\left(1, x_{2}, x_{3}\right)$ and an isotropic plane $A x+B y+$ $C z+D=0$ is given by

$$
\theta=\frac{\left|A+B x_{2}+C x_{3}\right|}{\sqrt{\left|B^{2}-C^{2}\right|}} .
$$

Therefore, the required angle is equal to

$$
\theta_{\alpha}=\frac{|\alpha k+\sigma|}{\sqrt{\left|\alpha^{2} k^{2}-1\right|}} .
$$

As in the Euclidean space, a surface with invariants $k=$ const, $\sigma=$ const, is called a surface of constant slope of type B. Now we have:

Theorem 2.6. The angle between the striction curve sof $\Phi$ and the asymptotic plane of the surface $\Phi_{\alpha}$ is constant if and only if $\Phi$ is either a surface of constant slope of type B, a torsal surface of constant slope or a conoidal surface of constant striction. That angle does not depend on $\alpha$ if and only if $\Phi$ is conoidal.

Proof. We have $\partial \theta(x, \alpha) / \partial x=0$, for all $\alpha \in \mathbf{R}$ if and only if

$\alpha^{3}\left(k^{3} \sigma^{\prime}-k^{2} k^{\prime} \sigma\right)+\alpha^{2}\left(k^{2} \sigma \sigma^{\prime}-k k^{\prime}-\sigma^{2} k k^{\prime}\right)-\alpha\left(k \sigma^{\prime}+k^{\prime} \sigma\right)-\sigma \sigma^{\prime}=0$.

For $\alpha=0$ we can immediately conclude that $\sigma=$ const, which substituted in (2.7) gives

$$
k^{2} k^{\prime} \sigma=0, \quad k k^{\prime}+\sigma^{2} k k^{\prime}=0, \quad k^{\prime} \sigma=0 .
$$

Therefore, if $\sigma=0$, then $k=$ const $\neq 0$, which characterizes the torsal surfaces of constant slope.

If $\sigma=$ const $\neq 0$, then either $k=$ const $\neq 0$, which means that $\Phi$ is a surface of constant slope of type $\mathrm{B}$, or $k=0$, which gives conoidal surfaces.

The angle $\theta_{\alpha}$ does not depend on $\alpha$ if and only if $k=0$. 


\section{3. $\beta$-Transversal Surfaces}

A $\beta$-transversal surface $\Phi_{\beta}$ is a ruled surface obtained from $\Phi$ by rotating a ruling $\mathbf{e}_{1}$ of $\Phi$ in the normal plane $\nu$ about a striction point $\mathbf{s}(x)$ for a fixed angle $\beta$. The normal plane $\nu$ is spanned by the central normal vector $\mathbf{e}_{2}$ and the central tangent vector $\mathbf{e}_{3}$, which are isotropic vectors, and therefore it is a pseudo-Euclidean plane. Hence a ruling $\overline{\mathbf{e}}$ of $\Phi_{\beta}$ can be described as

$$
\overline{\mathbf{e}}=\operatorname{ch} \beta \mathbf{e}_{3}+\operatorname{sh} \beta \mathbf{e}_{2} .
$$

$\beta$-transversal surfaces $\Phi_{\beta}$ are ruled surfaces of type III, having isotropic rulings which are parallel to a pseudo-Euclidean plane. The function

$$
\tau_{\beta}=\frac{\bar{e}_{2}^{\prime}}{\bar{e}_{3}}=\frac{\bar{e}_{3}^{\prime}}{\bar{e}_{2}}
$$

is the only invariant of ruled surfaces of type III. By using the Frenet's formulas for the surface $\Phi$ (which is of type I), we get

$$
\tau_{\beta}=\tau, \quad \beta \in \mathbf{R} .
$$

Furthermore, it is easy to see the following:

Theorem 3.1. Ruled $C^{3}$-surfaces $\Phi$ and $\Phi_{\beta}$ intersect each other along $s$ under the angle $\beta$.

Proof. Since a tangent plane of $\Phi_{\beta}$ along $s$ is given by

$$
\sigma \operatorname{sh} \beta X+\operatorname{ch} \beta Y-\operatorname{sh} \beta Z=0
$$

by using (2.5) we get $\varphi=\beta$.

The ruled surface which has the central tangents $\mathbf{e}_{3}$ of $\Phi$ for the generators is the special case of $\beta$-transversal surfaces with $\beta=0$. It is therefore also a ruled surface of type III, its torsion is equal to the torsion of $\Phi$ and by Theorem 3.1 it is tangent to $\Phi$ along $s$. Similarly, the ruled surface of central normals $\mathbf{e}_{2}$ of $\Phi$ is a ruled surface of type III, but unless we introduce complex angles, it is not a special case of $\beta$-transversal surfaces. Its torsion is equal to the torsion of $\Phi$, but the angle as in Theorem 3.1 is not defined.

Contrary to the situation in the Euclidean and the isotropic space, since surfaces $\Phi_{\beta}$ are surfaces of type III, and therefore without a striction curve, the analogous results to those for the surfaces $\Phi_{\alpha}$ cannot be obtained.

\section{4. $\gamma$-Transversal Surfaces}

Finally, let us define $\gamma$-transversal surfaces. The central plane $\zeta$ of $\Phi$ is a plane in the striction point $\mathbf{s}(x)$ spanned by the generator $\mathbf{e}_{1}$ and 
the central tangent vector $\mathbf{e}_{3}$. Because $\mathbf{e}_{1}$ is non-isotropic and $\mathbf{e}_{3}$ isotropic, the plane $\zeta$ is an isotropic plane. We define $\overline{\mathbf{e}}$ as the vector in $\zeta$ that forms a constant isotropic angle $\gamma \in \mathbf{R}$ with $\mathbf{e}_{1}(x)$. Hence,

$$
\overline{\mathbf{e}}=\mathbf{e}_{1}+\gamma \mathbf{e}_{3} .
$$

A $\gamma$-transversal surface $\Phi_{\gamma}$ is the ruled surface whose rulings are straight lines through a striction point $\mathbf{s}(x)$ determined by $\overline{\mathbf{e}}$.

We assume that the vector $\overline{\mathbf{e}}^{\prime}=(\kappa+\gamma \tau) \mathbf{e}_{2}$ is not the zero-vector, which gives the condition $\kappa+\gamma \tau \neq 0$, i.e., $\Phi$ is not a surface of constant slope equal to $-1 / \gamma$.

The following theorem which was considered already by K. GOROWARA in $E^{3}$ holds in $I_{3}^{1}$ [7] as well as in $G_{3}^{1}$ :

Theorem 4.1. The striction curve $s$ of a ruled surface $\Phi$ is the striction curve of a surface $\Phi_{\gamma}, \gamma \in \mathbf{R}$ as well. The surfaces $\Phi$ and $\Phi_{\gamma}$ are tangent to each other along $s$.

Proof. The striction curve on $\Phi_{\gamma}$ is determined from the condition

$$
v=\frac{\left(\tilde{\overline{\mathbf{e}}}-\tilde{\mathbf{s}}^{\prime}\right) \cdot \tilde{\overline{\mathbf{e}}}^{\prime}}{\left|\overline{\mathbf{e}}^{\prime}\right|^{2}} .
$$

Since $\mathbf{s}^{\prime}=\mathbf{e}+\sigma \mathbf{e}_{3}$ by some calculation we get $v=0$. Furthermore, tangent planes of $\Phi$ and $\Phi_{\gamma}$ along $s$ are given by $Y=0$.

We can notice now that $\Phi_{\gamma}$ is a surface of type I if and only if $\Phi$ is of type I.

Furthermore, from the previous theorem it follows immediately that the central normal surfaces $\Phi^{n}$ and $\Phi_{\gamma}^{n}$ of $\Phi$ and $\Phi_{\gamma}$ coincide, and therefore we have the analogue of the Euclidean result:

Theorem 4.2. Every surface $\Phi$ satisfies $\delta\left(\Phi_{\gamma}^{n}\right)=\delta\left(\Phi^{n}\right), k\left(\Phi_{\gamma}^{n}\right)=$ $k\left(\Phi^{n}\right)$, for every $\gamma \in \mathbf{R}$.

The following theorem holds in the Euclidean space as well, whereas in the simply isotropic space it holds for a different class of surfaces:

Theorem 4.3. The only ruled $C^{3}$-surfaces whose all $\gamma$-transversal surfaces are of constant parameter of distribution are ruled screw surfaces and $K$-surfaces. That constant parameter of distribution always depends on $\gamma$.

Proof. The parameter of distribution $\delta_{\gamma}$ can be written as

$$
\delta_{\gamma}=\frac{\sigma-\gamma}{\kappa+\gamma \tau} .
$$


We have $\partial \delta(x, \gamma) / \partial x=0$, for all $\gamma \in \mathbf{R}$, if and only if

$$
\gamma^{2} \tau^{\prime}+\gamma\left(\sigma^{\prime} \tau-\sigma \tau^{\prime}+\kappa^{\prime}\right)+\sigma^{\prime} \kappa-\sigma \kappa^{\prime}=0 .
$$

It follows immediately $\delta=$ const, and

$$
\tau^{\prime}=0, \quad \sigma^{\prime} \tau+\kappa^{\prime}=0 .
$$

The second condition $\kappa^{\prime}=-\sigma^{\prime} \tau$ of (4.8) substituted in $\delta^{\prime}=0$ gives $\sigma^{\prime}(\kappa+\sigma \tau)=0$. Therefore, either it follows $\sigma=$ const, which with $\delta=$ const and the first condition in (4.8) describes ruled screw surfaces or $\kappa+\sigma \tau=0$, which describes $K$-surfaces.

Theorem 4.4. $\gamma$-transversal surfaces of $K$-surfaces are also $K$-surfaces.

Proof. If $\Phi$ is a $K$-surface, then $\delta^{\prime}=0, \delta \tau+1=0$. The last condition implies $\tau^{\prime}=0$ and therefore, as in the proof of the previous theorem, we can conclude that $\delta_{\gamma}^{\prime}=0, \gamma \in \mathbf{R}$. Furthermore, because $s$ is asymptotic on $\Phi$ and $\Phi$ and $\Phi_{\gamma}$ are tangent along $s$, then $s$ is asymptotic on $\Phi_{\gamma}$ as well. Therefore $\Phi_{\gamma}$ is a $K$-surface.

Since the conical curvature of a $\gamma$-transversal surface $\Phi_{\gamma}$ is equal to

$$
\left|1 / k_{\gamma}\right|=|1 / k+\gamma| \text { for } k \neq 0, \quad k_{\gamma}=0 \quad \text { for } k=0,
$$

it is obvious that the following theorem holds:

Theorem 4.5. The only ruled $C^{3}$-surfaces whose all $\gamma$-transversal surfaces are surfaces of constant slope (conoidal surfaces) are surfaces of constant slope (conoidal surfaces). The slope always varies with $\gamma$.

The angle between two isotropic non-light planes $A_{i} X+B_{i} Y+$ $C_{i} Z+D_{i}=0, B_{1}: C_{1}=B_{2}: C_{2}, B_{i}^{2}-C_{i}^{2} \neq 0, i=1,2$, is equal to (see [1])

$$
\varphi=\frac{\left|A_{2}-A_{1}\right|}{\sqrt{\left|B_{1}^{2}-C_{1}^{2}\right|}},
$$

and therefore the angle between the asymptotic planes $Z=0$ of $\Phi$ and $\gamma X-Z=0$ of $\Phi_{\gamma}$ is equal to $\pm \gamma$.

Finally, by using (2.6) it is easy to show that the angle between the striction curve $s$ of $\Phi$ and the asymptotic plane of $\Phi_{\gamma}$ is equal to $\varphi_{\gamma}=|\gamma+\sigma|$. Therefore:

Theorem 4.6. The angle between the striction curve s of $\Phi$ and the asymptotic plane of $\Phi_{\gamma}$ is constant if and only if $\Phi$ is a surface of constant striction. 


\section{References}

[1] DivjaK, B. (1997) Geometrija pseudogalilejevih prostora. Ph. D. Thesis, University of Zagreb

[2] DivJAK, B., MiLIN ŠIPUŠ, Ž. (2003) Special curves on ruled surfaces in Galilean and pseudo-Galilean space. Acta Math. Hungar. 98(3): 203-215

[3] DIVJAK, B., MILIN ŠIPUŠ, Ž. (2003) Minding isometries of ruled surfaces in pseudo-Galilean space. J. Geom. 77: 35-47

[4] KRUPPA, E. (1949) Zur Differentialgeometrie der Strahlflächen und Raumkurven. Sitzungsber. Akad. Wiss. Wien 157: 143-176

[5] Röschel, O. (1984) Die Geometrie des Galileischen Raumes. Habilitationsschrift, Leoben

[6] SACHS, H. (1978) Über Transversalflächen von Regelflächen. Sitzungsber. Akad. Wiss. Wien 186: 427-439

[7] TAOUKTSOGLOU, A. (1994) Transversalflächen von Regelflächen im einfach isotropen Raum. Sitzungsber. Akad. Wiss. Wien 203: 137-148

Authors' addresses: Dr. Željka Milin Šipuš, Department of Mathematics, University of Zagreb, P.P. 335, 10002 Zagreb, Croatia, E-Mail: milin@math.hr; Dr. Blaženka Divjak, Faculty for Organisation and Informatics, University of Zagreb, Pavlinska 2, 42000 Varaždin, Croatia, E-Mail: bdivjak@foi.hr. 